\title{
Centralized Smart Parking and Insurance Applications for Intelligent Vehicles in a Smart City Utilizing the Cloud Computing Paradigm
}

\author{
Elmer R. Magsino \\ Electronics and Communications Engineering Department, Gokongwei College of Engineering, De La Salle \\ University, Manila, Philippines, elmer.magsino@dlsu.edu.ph
}

\begin{abstract}
Vehicles and infrastructure in an Intelligent Transportation System (ITS) may communicate to improve travel convenience and reduce the vehicular troubles. One of the significant activities of car owners is searching for free space parking. The time spent by drivers for available slots diminishes their productivity and contributes to the degradation of the environment. In this work, preliminary results surveying cloud-based applications centering on centralized parking and insurance reporting by utilizing empirical mobility traces are presented. Mobility traces represent movements of future intelligent vehicles as they traverse an urban city. From the initial simulation findings, centralized smart parking is very feasible and will rely on whether the ITS will provide enough infrastructure to allow centralized requesting of parking slots.
\end{abstract}

Key words: Cloud-based applications, Smart Parking, Vehicle Insurance, Vehicular Networks.

\section{INTRODUCTION}

Intelligent Transportation Systems (ITS) rely on the understanding and analysis of vehicular movements and deployment of static and mobile roadside infrastructure to achieve transportation objectives such as reduced travel time, convenient travel journey, and immediate emergency response. [1].Autonomous vehicles [2] and infrastructure will exchange relevant road data information to achieve these objectives.

One of the critical necessities in traveling in urban cities is theneed to find a parking space without consuming too much timein locating one suitable spot. Most cities have only a limited number of these parking lots, and these spots are taken basedon a first-come, first-served basis. Intelligent parkingapplications involve searching, locating, and bidding forvacant slots or reserving occupied and highly demanded parking slots. These applications consider several criteriaset by the driver, such as parking proximity and fee, and length of
stay.Finding a suitable parking space can be time-consumingand energy-wasting and contribute to traffic jams. Mostresearch works have focused on standalone infrastructures indetermining occupancy by using sensors and detectionalgorithms [3, 4], then displaying totalavailable parking slots on their establishment's billboard. Some useRoadside Units(RSUs)for allotting parking spaces [5, 6].In [7], an intelligentcar parking system that integrates the sensor,communication, and application layers has beendeployed and tested in a university ground. The middleware provides the bestservices, such asparking lots locator and supervision, and route planning. A case study using context information such astraffic and road conditions and decision making of trafficauthorities is presentedin [8]to manage parking services dynamically. Parking Service Provider (PSP), a work inprogress, is another proposed business model that allows private parking owners to have theirlot listed in the cloud directory of available parking spaces[9].

Another convenience that can be integrated into ITS is the on-the-spot and real-time reporting of maintenance and road accidents for insurance claims and immediate road repair. Based on the situation, insurance companies can now use vehicular environment data to adjust and approve premiumsbased on the vehicular usage, such as speeding and brakinghabits, road conditions, time anddate of travel. This is termedas usage-based insurance (UBI) [10]. These collected data areutilized for the dynamic creation of insurance policies [11].Since there is a real-time datadelivery, and insurance trackingpolicy is viable that may allow drivers to receive incentives ordisincentives [12].

In this work, we present a survey on the possible deployment of cloud-computing applications based on the empirical mobility traces. This study focuses on a cloud-based centralized smart parking system and insurance and maintenance reporting. During its journey, intelligent vehicles can connect to the cloud server for parking slot reservation in their destination. If unavoidably possible, when these autonomous vehicles also need repair or assistance, real-time reporting can be implemented. There are two ways for an intelligent vehicle to connect to The Cloud, either 1) via 
roadside units (free/minimal charges), and 2) cellular network (provider-based charging).

The paper is outlined as follows: Section 2 discusses the centralized cloud-based applications and its deployment in an urban area. Section 3 shows the initial simulation results gathered from empirical mobility traces assuming that these traces are representative of the actual intelligent and autonomous vehicles. Section 4 concludes the work and presents future direction for further improving the research topic.

\section{CENTRALIZED VEHICULAR CLOUD-BASED APPLICATIONS IN A SMART CITY}

In this section, we provide the basic definition of cloud computing and explore two possible scenarios, namely, (1) smart parking, and (2) scheduled automobile insurance and maintenance, where cloud computing can be integrated into a smart city. These use-case scenarios are two of the essential obligations when having vehicles.

\subsection{Preliminaries of Cloud Computing Applied in a Vehicular Network}

Cloud computing is a recent computing development where computational resources such as powerful processors with massive storage are leased by users over the Internet to perform intensive processes and for safekeeping following an on-demand approach [13]. Cloud computing relies on a shared set of resources for accomplishing a specific task with minimal supervision at a faster and ubiquitous approach [14]. Currently, the cloud computing paradigm has been used in business, education, and financial industries [15].

Consider the case of a cloud computing application scenario depicted in Figure 1. The simplest example is for monitoring traffic and road conditions. Vehicles can communicate among themselves and road infrastructure, such as roadside units (RSUs) via Dedicated Short-Range Communication (DSRC) to obtain current road situations. Vehicles can also directly contact The Cloud using 4G/LTE/cellular communication if they want to obtain surrounding conditions from faraway road segments. If a vehicle requests via local RSUs, the request is forwarded to a VANET Control Center (VCC). The VCC checks if the request is available in its database; otherwise, it sends the request to The Cloud before it can send the requested data to the requesting vehicle. The VCC also performs requests aggregation before sending it to The Cloud. By accessing The Cloud (via RSU or cellular communications), vehicles now have a broader perspective of their surroundings; therefore, they are now capable of making better judgments and decisions.

Cloud-based vehicular applications may take various types of connections to The Cloud, i.e., (1) always, (2) standalone, and (3) on-demand. Always connected requires vehicles to have access to The Cloud all the time. This always-connected is achieved by having a dedicated $4 \mathrm{G} / \mathrm{LTE}$ connection installed in the vehicle, and access to information is available all the time. On the other hand, standalone only connect to The Cloud if there is network access available. Lastly, on-demand communicates to The Cloud when the necessity arises. Among these three types of connections, the on-demand connection is best suited for smart parking and automobile maintenance and insurance applications. Intelligent vehicles will only connect to The Cloud when they need to book an advanced parking slot, or worse when the intelligent vehicle is involved in an accident and needs to be fixed or file an accident report. Also, smart parking and automobile maintenance and insurance are not data- and computational intensive services for The Cloud, unlike information dissemination that may require fog nodes in between the vehicles and The Cloud [17].

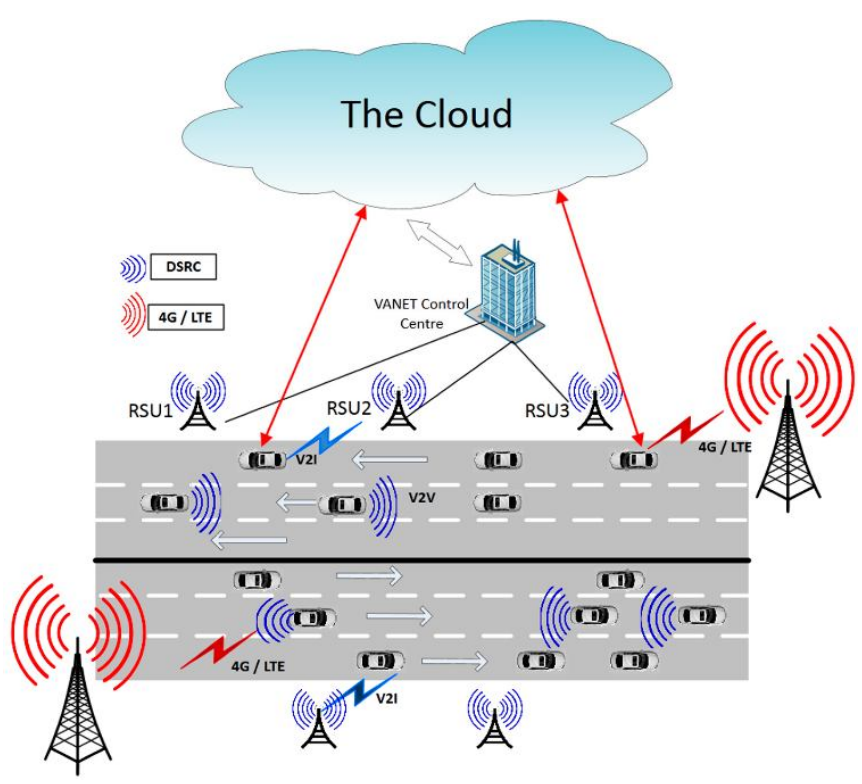

Figure 1:Vehicular network incorporating cloud computing for implementing smart city applications[16].

\subsection{Network Model for Smart Parking and Automobile Insurance}

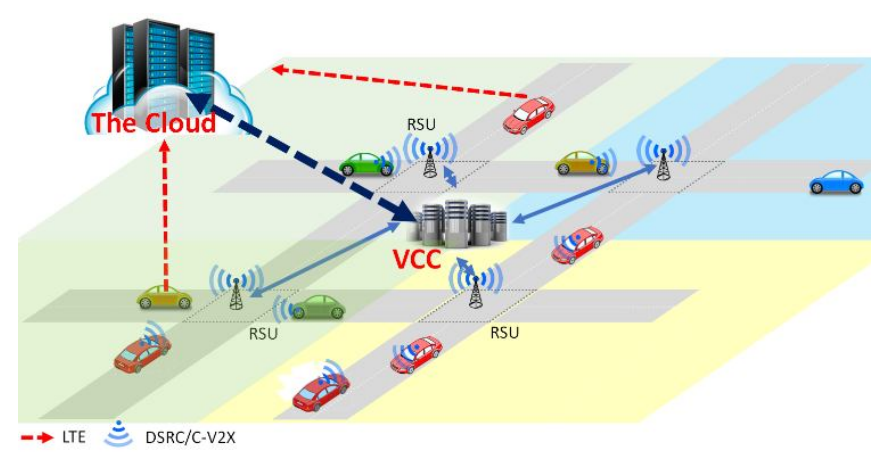

Figure 2: An urban map divided into regions according to the average vehicular density. 
Consider an urban city as depicted in Figure 2, where the partitioning is based on an area's vehicular density that is less than or equal to a threshold value, $Q_{\alpha}$. Each map partition has a roadside unit deployed in an intersection where vehicles can communicate in case they do not have $4 \mathrm{G} / \mathrm{LTE}$ access directly to The Cloud. We also assume that each region has only enough parking space for $50 \%$ of the region's average vehicular density. In Los Angeles, only $14 \%$ of its incorporated land is dedicated to parking [18].

\subsection{Cloud-based Parking Slot Reservation}

The process of availing a reserved parking space or reporting maintenance assistance in a vehicle's region of destination are enumerated below.

1. A vehicle sends a parking request via local RSU/Cellular, depending on its location. If the vehicle reserves via its local RSU, it pays nothing. However, when the vehicle uses the cellular network, it is charged by a reservation fee, $R_{f e e}$.

2. If the request is made via local RSU, there are two possible scenarios.

a. If the parking slot is also in the region of the requestor, the local RSU reserves a slot if there is available space. The local RSU automatically notifies the requestor and the VCC regarding a locally reserved parking slot(s), and in turn, the VCC will notify The Cloud.

b. Suppose the parking request is for another region, the local RSU checks for other off-region requests and aggregates them to a single report. It immediately forwards theaggregated request to the VCC for reservation. The local RSU will notify the requestor if there is a successful parking reservation, as instructed by the VCC.

The local RSU collates all $v_{l}(t)$ requests from $V$ vehicles at time tinto (1) and sendsit to the VCC.

$$
R_{R S U}(t) \square \bigcup_{l=1}^{V} v_{l}(t)
$$

The VCC then collects all $R_{R S U}$ 's coming from $j$ infrastructure at time $t$ and combines them to (2), and sendsit to The Cloud.

$$
R_{V C C}(t) \square \bigcup_{j=1}^{J} R_{R S U j}(t)
$$

3. If the request is made via cellular transmission, then The Cloud will inform the requesting vehicle and respective VCC if there is an available parking slot. The affected VCC then notifies the concerned local RSU to update its database.

4. The reservation is only valid for an interval, $T_{p a r k}$. If the vehicle does not arrive on its reserved parking space, The Cloud, VCC, and concerned local RSU vacates the slot and will assign it to the next requestor.

We assume that the parking spaces allotted per region are the targeted spaces of the requesting vehicles, regardless of how far it is from the desired parking lot of the requestor. This assumption holds since we do not have enough data to determine the location of the region's specific parking spaces. The VANET Control Center also resolves reservation conflicts based on a first-come, first-served basis. If there are two or more simultaneous requests, then the priority is chosen randomly.

\subsection{Automobile InsuranceReport}

Unlike the parking reservation procedure, the vehicle directly conveys its automobile insurance report to The Cloud, since insurance companies are generally located in fewer places than parking lots. More importantly, the vehicle involved in an accident must quickly submit the requirements and its vehicular data to the insurance company for immediate processing. Therefore, car insurance automatically incurs communications charges.

\section{SIMULATION RESULTS AND DISCUSSION}

In this section, we present simulation results utilizing the empirical mobility traces coming from the City of Beijing [19]. The dataset contains a seven-day mobility traces of approximately 28,000 taxis plying the City of Beijing and will be used to represent the mobility coordinates of intelligent vehicles.

\subsection{Empirical Mobility Dataset}

Figure 3 illustrates the area of Beijing City, where the cloud-based vehicular applications will be surveyed. We divide the region under study into four partitions and select 40 intersections where to place the local RSUs. The GPS coordinates of each intelligent vehicle are sampled every two minutes to capture the movement of the vehicles accurately.

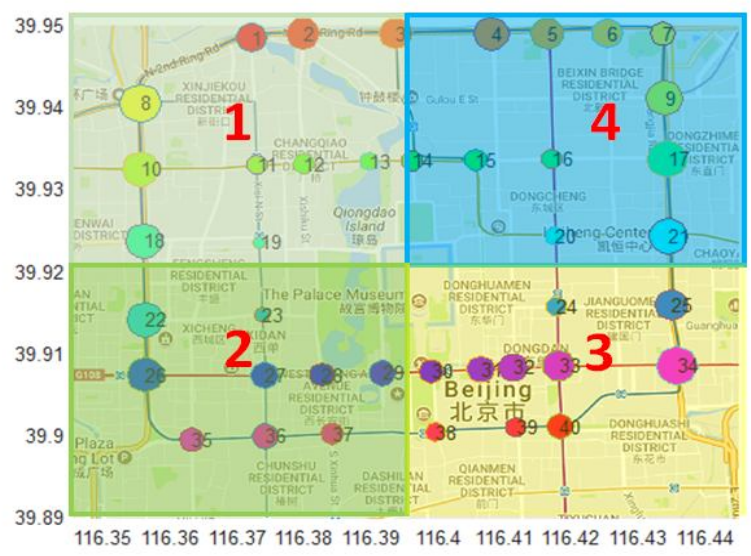

Figure 3: The urban map is divided into four monitoring regions. Each division has approximately ten intersections. 


\subsection{Evaluation of Cloud-based Centralized Smart Parking Applications in a Smart City}

From the given plan trip of each vehicle in the dataset traversing the region under study, the origin-destination pairs are extracted. We assumed that the destination automatically corresponds to the region (either 1, 2, 3, or 4) where they would want to look for a parking slot, and the conditions for parking reservations enumerated in Section 2 are followed.

Figure 4 illustrates the vehicular distribution whose destination corresponds to region $x$, where $x=1,2,3$, or 4 . The blue bar denotes the vehicles having their origin and destination regions identical, while the orange bar indicates vehicles coming from other regions. If only $50 \%$ of these vehicular distributions can be served parking slots per region, a centralized smart parking application can be beneficial to all intelligent and autonomous vehicles. Such an application will allow users to learn current vacant slots or which slots will be vacant in the next few minutes.

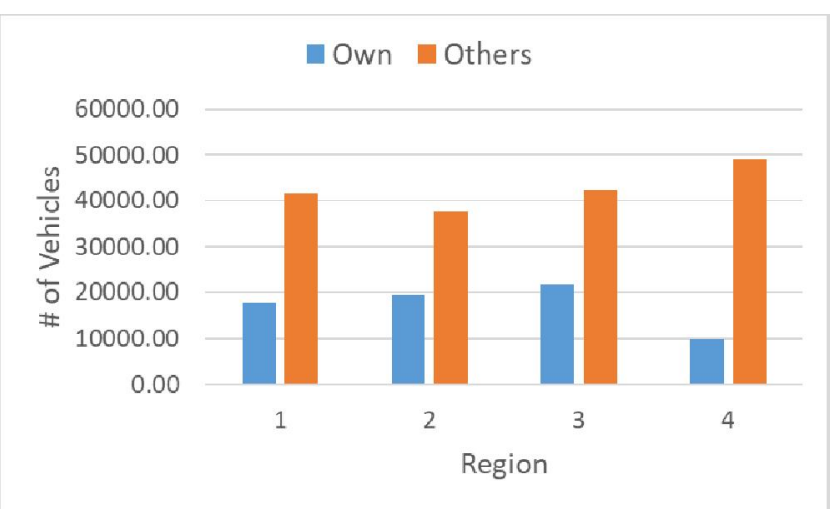

Figure 4: Number of vehicles which are parking in Region $\mathrm{x}$.

Assuming that at each sampling time and during its travel plan, the intelligent autonomous vehicle sends an inquiry to The Cloud via RSU or cellular network, the total number of RSU and cellular requests is shown inFigure 5. Practically, these numbers present the worst-case request scenario. Specifically, for the cellular requests, these numbers will go down once the intelligent autonomous vehicle considers the cellular costs. Also, this request distribution depicts the need to deploy more RSUs further so that the vehicles will less rely on the cellular network. It should be targeted that all requests shall pass only one mode of transmission to ensure more straightforward and more compact processing at The Cloud. Figure 5 also reveals that only vehicle-to-infrastructure (V2I) communications are the only actual means.

Since there are more cellular transactions than local RSU activities, intelligent vehicles are directly contacting The Cloud for their parking reservations. However, this can flood The Cloud and may result in delayed responses. If autonomous vehicles can communicate with RSUs more often, then it is possible to achieve a localized and decentralized parking reservation system and will reduce the inquiry burdens at The Cloud and VCC.

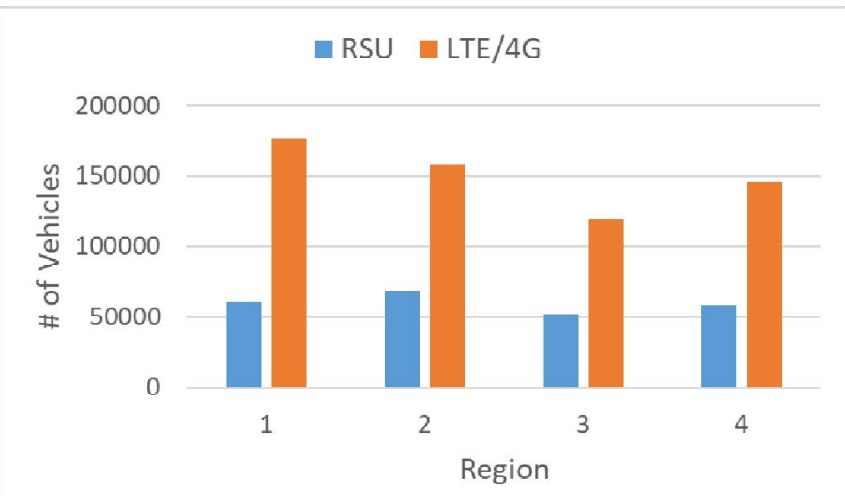

Figure 5:Average Daily Number of RSU vs. Cellular requests per region.

\subsection{Insurance/Maintenance Real-time Reporting}

Unlike the centralized smart parking application, the insurance/maintenance reporting is achieved by assuming that when a vehicle is at the same location during three consecutive sampling times. We note that, given this assumption, about $20 \%$ of the mobility traces satisfy the criterion. However, we may note here that in actual cases, this criterion may be caused by traffic congestions only.

If the UBI method is used to quantify the damages and compensation amount, then intelligent autonomous vehicles are automatically asked to forward their vehicular log data to The Cloud. If maintenance is asked, then The Cloud dispatches an emergency signal to the maintenance provider nearest the requesting vehicle.

\section{CONCLUSION}

In this work, we have presented preliminary results on the feasibility of a centralized smart parking andinsurance and maintenance reporting in an urban area based on the possible vehicular demand.Given that the demand is high, intelligent autonomous vehicles are advised to submit regional parking requests to The Cloud or via localized RSUs to reduce detrimental environmental effects and productivity hours. Intelligent autonomous vehicles can also send real-time and up-to-date travel $\log$ data to ensure correct insurance computations and on-time and fast maintenance road repair.

In the future, the centralized parking system will be developed and its components will be discussed in detail. From the system's perspective, another critical aspect that must be considered is the load balancing between local RSUs and VCC. Further research must consider the proper placements of these RSUs and VCC such that the workload and requests are almost equal for all infrastructure [20]. It is highly recommended that other urban cities be also surveyed to determine the prospect of the this topic. 


\section{ACKNOWLEDGEMENT}

This research work is supported by the University ResearchCoordination Office of De La Salle University, Manila, Philippines (30 F U 2TAY19-2TAY20).

\section{REFERENCES}

[1] G. Dimitrakopoulos and P. Demestichas, "Intelligent transportation systems,"IEEE Vehicular Technology Magazine, vol. 5, no. 1, pp. 77-84, 2010.

[2] G. P. T. Mayuga and E. R. Magsino, "Adaptive Cruise Control Employing Taillight Tracking for a Platoon of Autonomous Vehicles,"International Journal of Advanced Trends in Computer Science and Engineering, vol. 8, no. 3, pp. 640-645, 2019.

https://doi.org/10.30534/ijatcse/2019/48832019

[3] K. Choeychuen, "Automatic parking lot mapping for available parking space detection," in 2013 5th International Conference on Knowledge and Smart Technology (KST), 2013.

[4] C. T. M. Keat, C. Pradalier and C. Laugier, "Vehicle detection and car park mapping using laser scanner," in International Conference on Intelligent Robots and Systems, 2005.(IROS 2005). 2005 IEEE/RSJ, 2005.

[5] R. Panayappan, J. M. Trivedi, A. Studer and A. Perrig, "Vanet-based approach for parking space availability," in Proceedings of the fourth ACM international workshop on Vehicular ad hoc networks, 2007.

https://doi.org/10.1145/1287748.1287763

[6] R. Lu, X. Lin, H. Zhu and X. Shen, "Spark: a new vanet-based smart parking scheme for large parking lots," in INFOCOM 2009, 2009.

[7] Z. Ji, I. Ganchev, M. O'Droma, L. Zhao and X. Zhang, "A cloud-based car parking middleware for iot-based smart cities: Design and implementation,"Sensors, vol. 14, no. 12, pp. 22372-22393, 2014.

[8] J. Wan, D. Zhang, S. Zhao, L. Yang and J. Lloret, "Context-aware vehicular cyber-physical systems with cloud support: architecture, challenges, and solutions,"IEEE Communications Magazine, vol. 52, no. 8, pp. 106-113, 2014.

[9] Y. Atif, J. Ding and M. A. Jeusfeld, "Internet of things approach to cloud-based smart car parking,"Procedia Computer Science, vol. 98, pp. 193-198, 2016.

[10] P. Handel, M. Ohlsson, I. Skog and E. Nygren, "Smartphone-based measurement systems for road vehicle traffic monitoring and usage-based insurance,"IEEE Systems Journal, vol. 8, no. 4, pp. 1238-1248, 2014.

[11] S. T. Christensen, G. Hayward, C. E. Gay, S. Cielocha and T. Binion, "Dynamic auto insurance policy quote creation based on tracked user data". US Patent 9,141,996, 22 Sep 2015.
[12] C. P. Ricci, "Insurance tracking". US Patent 13/678,673, 16 Nov 2012.

[13] Q. Zhang, L. Cheng and R. Boutaba, "Cloud computing: state-of-the-art and research challenges,"Journal of internet services and applications, pp. 7-18, 2010.

https://doi.org/10.1007/s13174-010-0007-6

[14] P. Mell and T. Grance, "The NIST definition of cloud computing," 2011.

[15] O. Vaganova, Z. Smirnova, E. Vovk, A. Kapina and E. Chelnokova, "Comparative analysis of Cloud Technologies,"International Journal of Advanced Trends in Computer Science and Engineering, vol. 9, no. 3, pp. 2563-2566, 2020. https://doi.org/10.30534/ijatcse/2020/12932020

[16] K. Zaidi and M. Rajarajan, "Vehicular internet: security \& privacy challenges and opportunities,"Future Internet, vol. 7, no. 3, pp. 257-275, 2015.

[17] E. R. Magsino and I. W.-H. Ho, "Roadside Unit Allocation for Fog-based Information Sharing in Vehicular Networks," in Proceedings of the 1st ACM International Workshop on Smart Cities and Fog Computing, Shenzhen, China, 2018.

[18] M. Chester, A. Fraser, J. Matute, J. Flower and R. Pendyala, "Parking infrastructure: A constraint on or opportunity for urban redevelopment? A study of Los Angeles County parking supply and growth,"Journal of the American Planning Association 8, vol. 81, no. 4, pp. 268-286, 2015. https://doi.org/10.1080/01944363.2015.1092879

[19] I. W.-H. Ho, S. C. K. Chau, E. Magsino and K. Jia, "Efficient 3D Road Map Data Exchange for Intelligent Vehicles in Vehicular Fog Networks,"IEEE Transactions on Vehicular Technology, vol. 69, no. 3, pp. 3151-3165, 2020.

[20] N. Prasadh and M. Mehfooza, "An Efficient Hybrid Load Balancing Algorithm for Heterogeneous Data Centers in Cloud Computing,"International Journal of Advanced Trends in Computer Science and Engineering, vol. 9, no. 3, pp. 3078-3085, 2020. https://doi.org/10.30534/ijatcse/2020/89932020

[21] J. Liu, Z. Wu, J. Dong, J. Wu and D. Wen, "An energy-efficient failure detector for vehicular cloud computing,"PloS one, vol. 13, no. 1, 2018. 